\title{
Competitive CatSper Activators of Progesterone from Rhynchosia volubilis
}

\section{(우(1) $(2) \Theta$}

\section{Authors}

Jin Xiang ${ }^{1 *}$, Hang Kang ${ }^{2 *}$, Hong-Gang Li ${ }^{3 *}$, Yu-Long Shi ${ }^{5,6}$, Ya-Li Zhang ${ }^{4}$, Chang-Lei Ruan ${ }^{1}$, Lin-Hui Liu ${ }^{1}$, Han-Qi Gao ${ }^{1}$, Tao Luo ${ }^{2}$, Gao-Sheng Hu${ }^{4}$, Wei-Liang Zhu ${ }^{5,6}$, Jing-Ming Jia ${ }^{4}$, Jia-Chun Chen ${ }^{1}$, Jin-Bo Fang ${ }^{1}$

\section{Affiliations}

1 School of Pharmacy, Hubei Key Laboratory of Natural Medicinal Chemistry and Resource Evaluation, Tongji Medical College, Huazhong University of Science and Technology, Wuhan, China

2 Institute of Life Science and School of Life Science, Nanchang University, Key Laboratory of Reproductive Physiology and Pathology in Jiangxi Province, Nanchang, China

3 Institute of Reproductive Health/Center of Reproductive Medicine, Tongji Medical College, Huazhong University of Science and Technology, Wuhan, China

4 School of Traditional Chinese Materia Medica, Shenyang Pharmaceutical University, Shenyang, China

5 CAS Key Laboratory of Receptor Research \& Drug Discovery and Design Center, Shanghai Institute of Materia Medica, Chinese Academy of Sciences, Shanghai, China

6 School of Pharmacy, University of Chinese Academy of Sciences, Beijing, China

Key words

Leguminosae, Rhynchosia volubilis, CatSper, prenylated isoflavonoids, rhynchones A-E

received

February 22, 2021

accepted after revision

June 21, 2021

published online

August 6, 2021

Bibliography

Planta Med 2022; 88: 881-890

DOI $10.1055 / a-1542-0151$

ISSN 0032-0943

(c) 2021. The Author(s).

This is an open access article published by Thieme under the terms of the Creative Commons Attribution-NonDerivative-NonCommercial-License, permitting copying and reproduction so long as the original work is given appropriate credit. Contents may not be used for commercial purposes, or adapted, remixed, transformed or built upon. (https://creativecommons.org/licenses/by-nc-nd/4.0/)

Georg Thieme Verlag KG, Rüdigerstraße 14,

70469 Stuttgart, Germany
Correspondence

jin-Bo Fang

School of Pharmacy, Hubei Key Laboratory of Natural Medicinal Chemistry and Resource Evaluation, Tongji Medical College, Huazhong University of Science and Technology Hangkong Road 13, Qiaokou District, 430030 Wuhan, Hubei Province, China

Phone: + 862783692482 , Fax: +862783692482

fangjb@hust.edu.cn

$\notin$ Supplementary material is available under https://doi.org/10.1055/a-1542-0151

\section{ABSTRACT}

The root Rhynchosia volubilis was widely used for contraception in folk medicine, although its molecular mechanism on antifertility has not yet been revealed. In human sperm, it was reported that the cation channel of sperm, an indispensable cation channel for the fertilization process, could be regulated by various steroid-like compounds in plants. Interestingly, these nonphysiological ligands would also disturb the activation of the cation channel of sperm induced by progesterone. Therefore, this study aimed to explore whether the compounds in $R$. volubilis affect the physiological regulation of the cation channel of sperm. The bioguided isolation of the whole herb of $R$. volubilis has resulted in the novel discovery of five new prenylated isoflavonoids, rhynchones A-E (1-5), a new natural product, 5'-O-methylphaseolinisoflavan $(6)\left({ }^{1} \mathrm{H}\right.$ and ${ }^{13} \mathrm{C}$ NMR data, Supporting Information), together with twelve known compounds (7-18). Their structures were established by extensive spectroscopic analyses and drawing a comparison with literature data, while their absolute configurations were determined by electronic circular dichroism calculations. The experiments of intracellular $\mathrm{Ca}^{2+}$ signals and patch clamping recordings showed that rhynchone A (1) significantly reduced cation channel of sperm activation by competing with progesterone. In conclusion, our findings indicat that rhynchone A might act as a contraceptive compound by impairing the activation of the cation channel of sperm and thus prevent fertilization.

* These authors contributed equally to this study. 


\section{Introduction}

The genus Rhynchosia, belonging to the family Leguminosae, is composed of about 200 species, distributed in tropical and subtropical regions, but most of them are in Asia and Africa. There are 13 species in China, mainly distributed in the southern provinces of the Yangtze River [1]. The dry roots of Rhynchosia volubilis Lour. has shown diverse activities, including dispelling wind and dehumidification, promoting blood circulation, detoxification, detumescence, and relieving pain. It is also known as the king drug of a contraceptive prescription in folk medicine in clinics and has been used by natives in the northwest of Hubei Province, China, for female birth control for a long time [2]. The phytochemical investigations on this genus revealed the presence of flavonoids [3], isoflavonoids [4, 5], favan-3-ols, xanthones [6], biphenyls, simple polyphenols, and sterols [7]. Some of these exhibited antifertility [8], antimicrobial [9], antitumor [10], anti-inflammatory [11], antiproliferative [12], and antihyperlipidemic activities [13,14].

Calcium signaling in spermatozoa is essential for successful fertilization, which regulates the sperm capacitation, hyperactivation, and acrosome reaction $[15,16]$. The vital source of sperm intracellular free $\mathrm{Ca}^{2+}\left(\left[\mathrm{Ca}^{2+}\right]_{\mathrm{i}}\right)$ is the $\mathrm{Ca}^{2+}$ influx, predominantly mediated by the cation channel of sperm (CatSper), a pH-dependent voltage-gated $\mathrm{Ca}^{2+}$-selective channel $[17,18]$. CatSper is a highly complex multisubunit channel composed of at least ten subunits [19]: four separate pore-forming $\alpha$ subunits (CatSper 1-4) and six auxiliary subunits (CatSper $\beta, \gamma, \delta, \varepsilon, \zeta$, and EFCAB9). Mouse knockout models and genetic screening in infertile men demonstrated that CatSper is essential for male fertility in mice and humans [19]. In human sperm, the steroid hormones, progesterone (P4), prostaglandin (PG) E1, and PGE2, have been noted as potent CatSper agonists [20]. Moreover, structurally diverse endocrine-disrupting chemicals activate the sperm-specific CatSper channel and desensitize sperm for physiological CatSper ligands [21]. Therefore, the CatSper channel is a polymodal chemosensor in human sperm. All these results suggest that the CatSper channel is an ideal target for contraceptive. In order to define whether the compounds from $R$. volubilis disturb the physiological activation of the CatSper channel, we investigated the effects of the phytochemical constituents in the whole plant of $R$. volubilis on the regulation of CatSper.

\section{Results and Discussion}

Firstly, given that the CatSper channel mainly dominates $\mathrm{Ca}^{2+}$ influx in human sperm, the effect of different extracts from $R$. volubilis on intracellular $\mathrm{Ca}^{2+}\left(\left[\mathrm{Ca}^{2+}\right]_{\mathrm{i}}\right)$ signals were evaluated. The results showed that the petroleum ether (PE) extracts gave rise to a rapid $\left[\mathrm{Ca}^{2+}\right]_{i}$ elevation, while EtOAc and $n-\mathrm{BuOH}$ extracts failed to reproduce this effect (Fig. 44S, Supporting Information).

The PE and EtOAc fraction from the crude EtOH extract from the whole herb of $R$. volubilis was subjected to repeated chromatography procedures (silica gel, Toyopearl HW-40C, Sephadex LH20 , and semipreparative HPLC), leading to the isolation of five new prenylated isoflavonoids, rhynchones A-E (1-5), the structures of which were characterized by interpretation of their HRMS, 1D and 2D NMR, and electronic circular dichroism (ECD) data. Besides the five new compounds (1-5), a new natural product, 5'-O-methylphaseolinisoflavan (6) [22], together with twelve known compounds (7-18) were obtained and identified as tonkinensisol (7) [23], lupinifolinol (8) [24], cathayanon H (9) [25], cajanone (10) [5], prunetin (11) [26], isowighteone (12) [27], erythrinin B (13) [28], semilicoisoflavone B (14) [29], eriosemaone D (15) [30], formononetin (16) [31], puerarone (17) [32], and bidwillon C (18) [33] by comparison with literature values ( $\vee$ Fig. 1). Herein, the isolation, structure elucidation, and potential CatSper regulation activities of these isolated compounds are described in detail.

To further explore which kind of compound regulated the homeostasis of $\left[\mathrm{Ca}^{2+}\right]_{\mathrm{i}}, 18$ compounds $(1-18)$ from $R$. volubilis on $\left[\mathrm{Ca}^{2+}\right]_{i}$ of human sperm were assessed. Interestingly, only rhynchone A (1) from the PE extracts evoked a transient amplitude of a $\left[\mathrm{Ca}^{2+}\right]_{i}$ signal (Figs. 44S and 45S, Supporting Information). The results of patch-clamp recordings also manifested that rhynchone A amplified the monovalent current of human sperm, indicating that the elevation of the $\left[\mathrm{Ca}^{2+}\right]_{i}$ signal caused by rhynchone $A$ resulted from the activation of CatSper ( $\bullet$ Fig. 2). More importantly, subsequent studies found that the elevation of $\left[\mathrm{Ca}^{2+}\right]_{i}$ caused by P4 was suppressed by rhynchone $A$. The results of patch-clamp recordings on human sperm also manifested that rhynchone A compromised the activation of the CatSper channel elicited by P4 ( $\vee$ Fig. 3). Therefore, these findings suggested that rhynchone $A$ attenuated the physiological activities of P4 on the CatSper channel, and as a result, affected the function of human sperm. Compared to compound 10 , we speculated that the configuration of the B-ring and the substitution of a methoxyl group at C-4' played a vital role in activating CatSper.

\section{Structure elucidation}

Rhynchone $A$ (1), a pale-yellow solid, has a molecular formula of $\mathrm{C}_{26} \mathrm{H}_{28} \mathrm{O}_{6}$ based on HR-ESI-TOF-MS data (Fig. 1S, Supporting Information) with an $\mathrm{m} / \mathrm{z}$ ion of 435.1794 for [M $-\mathrm{H}]^{-}$(calcd. 435.1807). The presence of ${ }^{1} \mathrm{H}$ resonances at $\mathrm{H}-2 \mathrm{a}\left(\delta_{\mathrm{H}} 4.68,1 \mathrm{H}\right.$, $d d, J=4.8,11.7 \mathrm{~Hz}), \mathrm{H}-2 \mathrm{~b}\left(\delta_{\mathrm{H}} 4.84,1 \mathrm{H}, d d, J=4.1,11.9 \mathrm{~Hz}\right)$, and $\mathrm{H}-3\left(\delta_{\mathrm{H}} 3.93,1 \mathrm{H}, \mathrm{br} t, J=4.4 \mathrm{~Hz}\right)$, and corresponding oxymethylene and methine signals at $\delta_{\mathrm{C}} 69.3,44.9$ in its ${ }^{1} \mathrm{H}$ and ${ }^{13} \mathrm{C}$ NMR spectra ( $\vee$ Tables 1 and 2, Figs. 25 and 3S, Supporting Information), respectively, suggested the presence of an isoflavanone skeleton. Signals at $\delta_{\mathrm{H}} 11.94(1 \mathrm{H}, \mathrm{s})$ and $5.93(1 \mathrm{H}, \mathrm{s})$ corresponded to the $\mathrm{C}-5$ hydroxy group and $\mathrm{H}-8$, respectively, which showed an ortho-substitution in the A-ring. The ${ }^{1} \mathrm{H}$ NMR spectrum of 1 exhibited four methyl groups at $\delta 1.42,1.44\left(3 \mathrm{H}, \mathrm{s}, \mathrm{C}-2^{\prime \prime}\right), 1.66$, and $1.71\left(3 \mathrm{H}, s, \mathrm{C}-3^{\prime \prime \prime}\right)$, one methoxyl proton at $\delta 3.77(3 \mathrm{H}, \mathrm{s})$, and three olefinic protons at $\delta 5.48(1 \mathrm{H}, d, J=10.1 \mathrm{~Hz}), 6.56(1 \mathrm{H}, d$, $J=10.1 \mathrm{~Hz})$, and $5.23(1 \mathrm{H}, \mathrm{m})$, which indicated the presence of two isopropenyl groups. ${ }^{1} \mathrm{H}-{ }^{1} \mathrm{H}$ COSY (Fig. 6S, Supporting Information) correlations were observed for $\mathrm{H}-3^{\prime \prime} / \mathrm{H}-4^{\prime \prime}$ and $\mathrm{H}-2^{\prime \prime \prime} / \mathrm{H}$ $3^{\prime \prime \prime}$, indicating the connectivity of $\mathrm{C}-3^{\prime \prime}$ to $\mathrm{C}-4^{\prime \prime}$ and $\mathrm{C}-2^{\prime \prime \prime}$ to $\mathrm{C}-3^{\prime \prime \prime}$. The HMBC correlations (Fig. 4S, Supporting Information) $\mathrm{H}-3^{\prime \prime}$ to $\mathrm{C}-2^{\prime \prime}$ and $\mathrm{C}-6$; $\mathrm{H}-4$ " to $\mathrm{C}-6, \mathrm{C}-7, \mathrm{C}-2^{\prime \prime}$, and $\mathrm{C}-3^{\prime \prime} ; \mathrm{H}_{3}-2^{\prime \prime}$ to $\mathrm{C}-2^{\prime \prime}, \mathrm{C}-$ $3^{\prime \prime}$, and C-10 indicated that C-4" was attached to C-6, and C-2" was linked with $\mathrm{C}-7$ by an ether bond. The aromatic proton signals at $\delta 6.48\left(1 \mathrm{H}, \mathrm{s}, \mathrm{H}-3^{\prime}\right)$ and $7.17\left(1 \mathrm{H}, \mathrm{s}, \mathrm{H}-6^{\prime}\right)$ indicated that the $\mathrm{B}$ ring was $1^{\prime}, 2^{\prime}, 4^{\prime}, 6^{\prime}$ - tetrasubstituted. The HMBC correlations from $\mathrm{H}-3$ to $\mathrm{C}-1^{\prime}$, $\mathrm{C}-2^{\prime}$; $\mathrm{H}-2$ to $\mathrm{C}-1^{\prime} ; \mathrm{H}_{3}-4^{\prime}-\mathrm{OMe}$ to $\mathrm{C}-4^{\prime}$; $\mathrm{H}-3^{\prime}$ to 


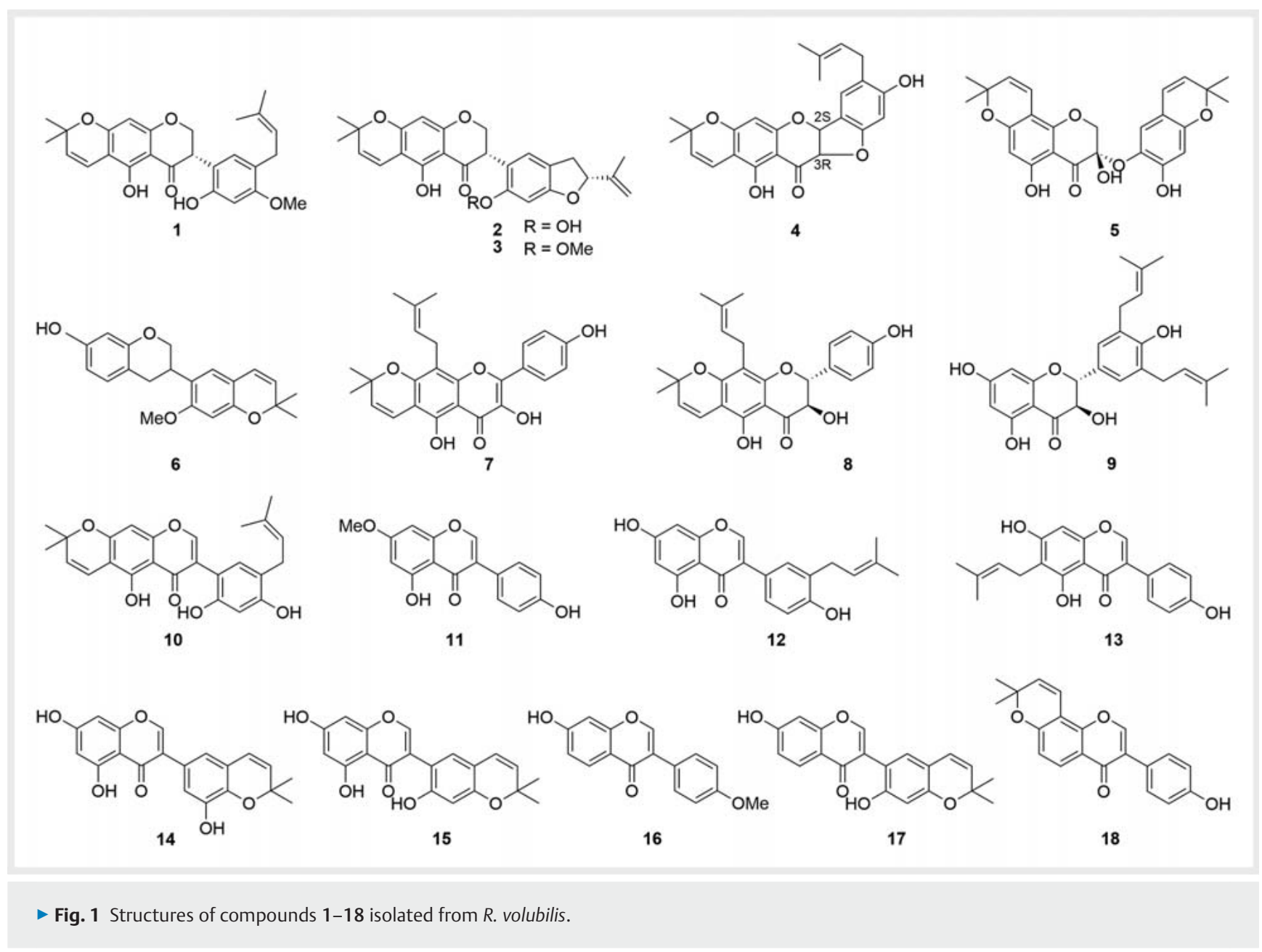

an oxidized aromatic quaternary $\mathrm{C}-2^{\prime}$; and $\mathrm{H}_{2}-1^{\prime \prime \prime}$ to $\mathrm{C}-4^{\prime}$ demonstrated the group substitution model in the B-ring ( $\triangleright$ Fig. 4 ). In order to determine the absolute configuration of 1 , a computational study using the time-dependent density functional theory (TD-DFT) method of ECD spectra at the B3LYP/6-31g (d, p) level was performed with Gaussian 16 B.01 [34]. Additionally, the solvent effects of methanol were taken into consideration with the integral equation formalism polarizable continuum model (IEFPCM) [35] during the calculations. The Boltzmann averaged spectra for all the possible conformers of 1 and their experimental ECD spectra are shown in - Fig. $\mathbf{5}$ a. The experimental ECD spectrum of 1 displayed high similarity to the calculated ECD pattern of 3S-1, which exhibited a calculated ECD spectrum with a distinct positive Cotton effect at $202 \mathrm{~nm}$ and a negative Cotton effect at $272 \mathrm{~nm}$ ( $\vee$ Fig.5a). Furthermore, a negative Cotton effect at $326 \mathrm{~nm}$ (Fig. 8S, Supporting Information) in the ECD spectrum of 1 also suggested the 35 configuration [36]. Thus, the structure of rhynchone A (1) was determined as 3S-5, 2', 4'-trihydroxy-2" , 2"dimethylpyrano $\left[6,7: 5^{\prime \prime}, 6^{\prime \prime}\right]-5^{\prime}$-prenyl-isoflavone.

Rhynchone $\mathrm{B}(2)$, a yellow oil, was deduced as having the molecular formula $\mathrm{C}_{26} \mathrm{H}_{28} \mathrm{O}_{6}$ by HR-ESI-TOF-MS [M $\left.+\mathrm{H}_{2} \mathrm{O}-\mathrm{H}\right]^{-} \mathrm{m} / \mathrm{z}$ 437.1606 (calcd. 437.1600), indicating one more index of hydrogen deficiency than 1 . The NMR spectroscopic data of 2 ( $\triangleright$ Tables 1 and 2 ) also showed structural similarity with 1 . The major differ- ence between these two compounds was found on the B-ring. The substitution at C-4' and C-5' was identified as an isopropenyl dihydrofuran group, which was characterized by the following: two endocyclic methylene protons, $\delta_{\mathrm{H}} 2.66(1 \mathrm{H}, d d, J=2.0$, $\left.14.6 \mathrm{~Hz}, 3^{\prime \prime \prime} \mathrm{a}\right)$ and $\left.2.87\left(1 \mathrm{H}, d d, J=8.6,14.9 \mathrm{~Hz}, 3^{\prime \prime \prime} \mathrm{b}\right)\right]$, two exocyclic methylene protons, $\delta_{\mathrm{H}} 4.86\left(\mathrm{H}, \mathrm{m}, 5^{\prime \prime \prime} \mathrm{a}\right)$ and $4.98\left(\mathrm{H}, \mathrm{m}, 5^{\prime \prime \prime} \mathrm{b}\right)$, an oxymethine signal, $\delta_{\mathrm{H}} 4.31(\mathrm{H}, t, J=8.0 \mathrm{~Hz}), \delta_{\mathrm{C}} 78.49\left(\mathrm{C}-2^{\prime \prime \prime}\right)$, and a methyl group $\left[\delta_{\mathrm{H}} 1.79\left(3 \mathrm{H}, \mathrm{s}, 6^{\prime \prime \prime}\right), \delta_{\mathrm{C}} 18.1\left(\mathrm{C}-6^{\prime \prime \prime}\right)\right]$. These were confirmed by ${ }^{1} \mathrm{H}-{ }^{1} \mathrm{H}$ COSY correlations (Fig. 14S, Supporting Information) for $\mathrm{H}-2^{\prime \prime \prime} / \mathrm{H}-3 \mathrm{~b}^{\prime \prime \prime}$, and $\mathrm{HMBC}$ correlations (Fig. 13S, Supporting Information) from $\mathrm{H}-2^{\prime \prime \prime}$ to $\mathrm{C}-5^{\prime} / 3^{\prime \prime \prime} / 5^{\prime \prime \prime} / 6^{\prime \prime \prime}$ and $\mathrm{H}-3 \mathrm{~b}^{\prime \prime \prime}$ to $\mathrm{C}$ $4^{\prime} / 5^{\prime} / 6^{\prime} / 2^{\prime \prime \prime} / 4^{\prime \prime \prime}$. The $S$ configuration of $\mathrm{C}-3$ was determined based on its circular dichroism spectrum (Fig. 16S, Supporting Information), and showed a negative cotton effect at $325 \mathrm{~nm}$ [36]. In the ROESY spectrum (Fig. 15S, Supporting Information), $\mathrm{H}-2^{\prime \prime \prime}\left(\delta_{\mathrm{H}}\right.$ 4.31) correlated with $\mathrm{H}_{2}-3^{\prime \prime \prime}\left(\delta_{H} 2.66,2.87\right), \mathrm{H}_{3}-6^{\prime \prime \prime}\left(\delta_{H} 1.79\right)$ correlated with $\mathrm{H}-2$ "', and the coupling constants of $\mathrm{H}-2^{\prime \prime \prime}$ and $\mathrm{H}-3 \mathrm{a}^{\prime \prime \prime}$ were different from those in crotadihydrofuran $\mathrm{A}$, which indicated that $\mathrm{H}-2^{\prime \prime \prime}$ was an $\beta$-orientation [37]. Thus, the structure of rhynchone B (2) was identified as 3S,2"'R-5,2'-dihydroxy-2"',2" - dimethylpyrano[6,7:5",6" $6^{\prime \prime}-2^{\prime \prime \prime}$-allyl furano[ $\left.4^{\prime}, 5^{\prime}: 4^{\prime \prime}, 5^{\prime \prime}\right]$ isoflavanone.

Rhynchone C (3), a yellow powder, had a molecular formula of $\mathrm{C}_{26} \mathrm{H}_{26} \mathrm{O}_{6}$ from its HR-ESI-TOF-MS spectra [M $\left.+\mathrm{H}_{2} \mathrm{O}-\mathrm{H}\right]^{-} \mathrm{m} / \mathrm{z}$ 451.1807 ([M + $\left.\mathrm{H}_{2} \mathrm{O}-\mathrm{H}\right]^{-}$calcd. 451.1757). The NMR data ( $\bullet \mathrm{Ta}-$ 

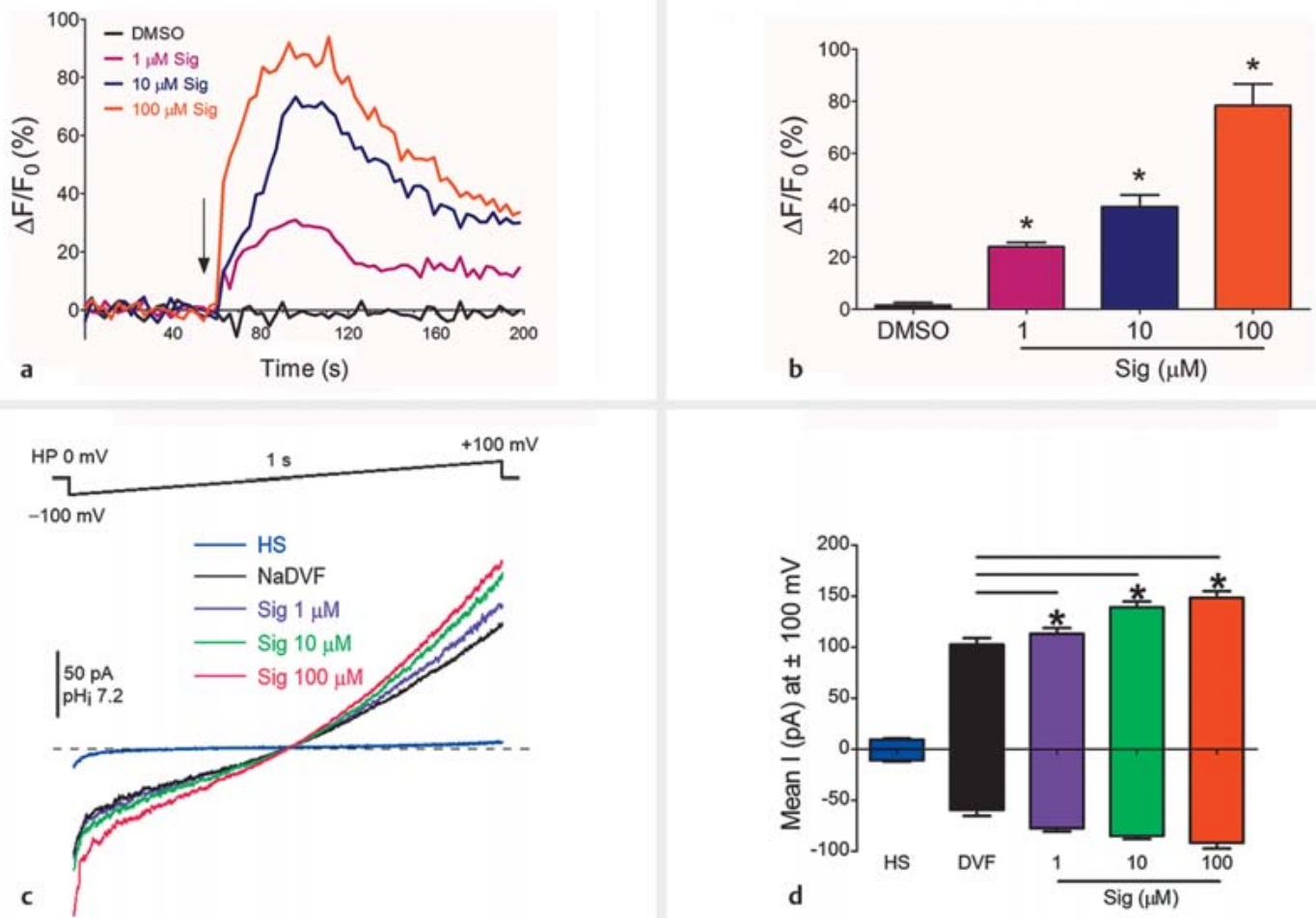

- Fig. 2 The effect of different concentrations of rhynchone A (1) on the activation of the CatSper channel of human sperm. a The typical fluorescence traces of $\left[\mathrm{Ca}^{2+}\right]_{i}$ signals before and after exposure to different concentrations of rhynchone $A(1)$. Arrow indicates the time point of additives in human sperm. b Average amplitudes of $\mathrm{Ca}^{2+}$ response in the presence of different concentrations of rhynchone $\mathrm{A}(1)$ are shown. $\mathbf{c}$ Representative monovalent current of human CatSper was potentiated by different concentrations of rhynchone A (1). The monovalent CatSper current was recorded in the presence of sodium-based divalent-free solution (NaDVF) by a voltage-clamp ramp protocol (from - $100 \mathrm{mV}$ to $+100 \mathrm{mV}, 1 \mathrm{~s}$ ). Holding potential (HP) was set to $0 \mathrm{mV}$. d Average currents of the CatSper channel at $-100 \mathrm{mV}$ (negative) and $+100 \mathrm{mV}$ (positive) after injecting different concentrations of rhynchone $A(1)$ are shown. Data are expressed as the mean $\pm S E M ; n=4,{ }^{*} p<0.05$.

bles 1 and 2) revealed a methoxy group $\left(\delta_{H} 3.79, \delta_{C} 55.5\right)$ instead of the $\mathrm{C}-2^{\prime}$ hydroxyl group in 2. This difference was demonstrated by the HMBC correlation from $\mathrm{H}_{3}-2^{\prime}$-OMe to $\mathrm{C}-2^{\prime}$ at $\delta_{\mathrm{C}} 158.4$ (Fig. 21S, Supporting Information). Thus, 3 was identified as 35, 2"'R-5-hydroxy-2'-methoxyl-2", 2"-dimethylpyrano [6,7:5",6"]$2^{\prime \prime \prime}$-allyl furano[ $\left.4^{\prime}, 5^{\prime}: 4^{\prime \prime}, 5^{\prime \prime}\right]$ isoflavanone.

Rhynchone D (4), a yellow oily solid, had a molecular formula of $\mathrm{C}_{25} \mathrm{H}_{24} \mathrm{O}_{6}$ based on its HR-ESI-MS ion at [M $\left.+\mathrm{H}_{2} \mathrm{O}-\mathrm{H}\right]^{-} \mathrm{m} / \mathrm{z}$ 437.1639 (calcd. 437.1600). The NMR spectra (Figs. 265 and 27S, Supporting Information) of 4 exhibited very similar A- and B-ring moieties with those of 1 . The $C-4$ ' was substituted by a hydroxyl group in $\mathbf{2}$ instead of a methoxy group in $\mathbf{1}$. Additionally, incorporating a furan ring in the flavone system, an extra ring was fused to ring $\mathrm{B}\left(\mathrm{C}-2-\mathrm{C}-3-\mathrm{O}-\mathrm{C}-2^{\prime}-\mathrm{C} 1^{\prime}\right)$. This assertion was supported by the ${ }^{1} \mathrm{H}-{ }^{1} \mathrm{H}$ COSY correlations of $\mathrm{H}-2\left(\delta_{\mathrm{H}} 4.72\right) / \mathrm{H}-3\left(\delta_{\mathrm{H}}\right.$ $4.32)$ (Fig. 30S, Supporting Information) and $\mathrm{HMBC}$ correlations from $\mathrm{H}-2\left(\delta_{\mathrm{H}} 4.72\right)$ to $\mathrm{C}-4\left(\delta_{\mathrm{C}} 194.8\right), \mathrm{C}-9\left(\delta_{\mathrm{C}} 162.4\right)$, and $\mathrm{C}-1^{\prime}\left(\delta_{\mathrm{C}}\right.$ $115.0)$ and $\mathrm{H}-3\left(\delta_{\mathrm{H}} 4.32\right)$ to $\mathrm{C}-4\left(\delta_{\mathrm{C}} 194.8\right)$ and $\mathrm{C}-1^{\prime}\left(\delta_{\mathrm{C}} 115.0\right)$ (Fig. 29S, Supporting Information). According to the coupling constant $(J=11.1 / 11.4 \mathrm{~Hz}$ ) between $\mathrm{H}-2 / \mathrm{H}-3$, we concluded that the two rings were trans-fused. In addition, the absolute configuration of 4 was approximate to $2 S, 3 R-4$, which was characteristic of the positive Cotton effects at 212 and $273 \mathrm{~nm}$ and the negative Cotton effect at $258 \mathrm{~nm}$ ( $\triangleright$ Fig. $\mathbf{5}$ b) in the ECD spectrum. The structure of rhynchone $D$ was deduced as $2 S, 3 R-5,4^{\prime}$-dihydroxy2", 2"-dimethylpyrano[6,7:5",6"] $]-5^{\prime}$-prenyl-furano $\left[2,3: 5^{\prime}, 4^{\prime}\right]$ flavonone.

Rhynchone $E$ (5), a yellow oily solid, had a molecular formula of $\mathrm{C}_{25} \mathrm{H}_{24} \mathrm{O}_{8}$ based on its HR-ESI-MS (Fig. 33S, Supporting Information) and NMR spectra (Figs. 345 and 35S, Supporting Information). The NMR data ( $\vee$ Tables $\mathbf{1}$ and 2 ) of substitution on its Aand $B$-rings resembled those of precatorin $A$, and the main difference between the two compounds was the connection between the $\mathrm{B}$ - and $\mathrm{C}$-rings. The molecular mass of rhynchone $\mathrm{E}$ (5) was 32 mass units higher than precatorin $A[4]$, indicating that 5 possessed a hemiacetalic carbon $\left(\delta_{C} 105.1, C-3\right)$. This was demonstrated by the following changes of carbon chemical shifts compared to precatorin A: C-2 $\left(\delta_{\mathrm{C}} 70.2,+0.5 \mathrm{ppm}\right), \mathrm{C}-4\left(\delta_{\mathrm{C}} 185.1\right.$, $+11.6 \mathrm{ppm}), \quad \mathrm{C}^{-1}{ }^{\prime} \quad\left(\delta_{\mathrm{C}} 140.6,+26.1 \mathrm{ppm}\right), \quad \mathrm{C}-2^{\prime} \quad\left(\delta_{\mathrm{C}} 146.6\right.$, -9.3 ppm), and $\mathrm{C}^{-6^{\prime}}\left(\delta_{\mathrm{C}} 105.9,19.2 \mathrm{ppm}\right)$ based on HSQC (Fig. 36S, Supporting Information), HMBC (Fig.37S, Supporting Information), and ${ }^{1} \mathrm{H}-{ }^{1} \mathrm{H}$ COSY (Fig. 38S, Supporting Information) analyses. The result of 5 showed that the experimental ECD spectrum exhibited a positive Cotton effect at $206 \mathrm{~nm}$ and a negative Cotton effect at $272 \mathrm{~nm}$, which was highly similar to the calculated ECD pattern of 3S-5 ( $\bullet$ Fig. 5 c). So, 5 was identified as 3,5dihydroxy-3-((7-hydroxy-2,2-dimethyl-2H-chromen-6-yl)oxy)8,8-dimethyl-2,3-dihydro-4H,8H-pyrano[2,3-f]chromen-4-one. 
- Table $1{ }^{1} \mathrm{H}$ NMR $\left(600 \mathrm{MHz}, \delta\right.$ in ppm, J in $\left.\mathrm{Hz}, \mathrm{CDCl}_{3}\right)$ data for compounds $1-5$.

\begin{tabular}{|c|c|c|c|c|c|}
\hline \multirow[t]{2}{*}{ Position } & 1 & 2 & 3 & 4 & 5 \\
\hline & $\delta_{\mathrm{H}}(/$ in $\mathrm{Hz})$ & $\delta_{\mathrm{H}}(/$ in $\mathrm{Hz})$ & $\delta_{\mathrm{H}}(/$ in $\mathrm{Hz})$ & $\delta_{\mathrm{H}}(J$ in $\mathrm{Hz})$ & $\delta_{\mathrm{H}}(/$ in $\mathrm{Hz})$ \\
\hline $2 a$ & $\begin{array}{l}4.68(1 \mathrm{H}, d d, J=4.8 \\
11.7 \mathrm{~Hz})\end{array}$ & $\begin{array}{l}\text { 4.62 (1H, dd, J=4.9, } \\
\text { 11.7 Hz) }\end{array}$ & $\begin{array}{l}4.70(1 \mathrm{H}, d d, J=4.6 \\
11.9 \mathrm{~Hz})\end{array}$ & $4.72(1 \mathrm{H}, d, J=11.1 \mathrm{~Hz})$ & $4.52(2 \mathrm{H}, \mathrm{s})$ \\
\hline $2 b$ & $\begin{array}{l}4.84(1 \mathrm{H}, d d, J=4.1 \\
11.9 \mathrm{~Hz})\end{array}$ & $\begin{array}{l}4.78(1 \mathrm{H}, \mathrm{dd}, J=5.9 \\
11.6 \mathrm{~Hz})\end{array}$ & $\begin{array}{l}4.85(1 \mathrm{H}, d d, J=5.7 \\
11.3 \mathrm{~Hz})\end{array}$ & & \\
\hline 3 & $3.93(1 \mathrm{H}, t, J=4.4 \mathrm{~Hz})$ & $4.03(1 \mathrm{H}, \mathrm{t}, J=5.3 \mathrm{~Hz})$ & $3.92(1 \mathrm{H}, \mathrm{m})$ & $4.32(1 \mathrm{H}, d, J=11.4 \mathrm{~Hz})$ & \\
\hline \multicolumn{6}{|l|}{4} \\
\hline \multicolumn{6}{|l|}{5} \\
\hline 6 & & & & & $5.98(1 \mathrm{H}, \mathrm{s})$ \\
\hline \multicolumn{6}{|l|}{7} \\
\hline 8 & $5.93(1 \mathrm{H}, \mathrm{s})$ & $5.93(1 \mathrm{H}, \mathrm{s})$ & $5.95(1 \mathrm{H}, \mathrm{s})$ & $5.91(1 \mathrm{H}, \mathrm{s})$ & \\
\hline \multicolumn{6}{|l|}{9} \\
\hline \multicolumn{6}{|l|}{10} \\
\hline \multicolumn{6}{|l|}{$1^{\prime}$} \\
\hline \multicolumn{6}{|l|}{$2^{\prime}$} \\
\hline $3^{\prime}$ & $6.48(1 \mathrm{H}, \mathrm{s})$ & $6.48(1 \mathrm{H}, \mathrm{s})$ & $6.52(1 \mathrm{H}, \mathrm{s})$ & $6.42(1 \mathrm{H}, \mathrm{s})$ & $6.42(1 \mathrm{H}, \mathrm{s})$ \\
\hline \multicolumn{6}{|l|}{$4^{\prime}$} \\
\hline \multicolumn{6}{|l|}{$5^{\prime}$} \\
\hline $6^{\prime}$ & $7.17(1 \mathrm{H}, \mathrm{s})$ & $7.00(1 \mathrm{H}, \mathrm{s})$ & $7.22(1 \mathrm{H}, \mathrm{s})$ & $6.64(1 \mathrm{H}, \mathrm{s})$ & $6.51(1 \mathrm{H}, \mathrm{s})$ \\
\hline \multicolumn{6}{|l|}{$2^{\prime \prime}$} \\
\hline $3^{\prime \prime}$ & $5.48(1 \mathrm{H}, d, J=10.1 \mathrm{~Hz})$ & $5.49(1 \mathrm{H}, d, J=10.1 \mathrm{~Hz})$ & $5.48(1 \mathrm{H}, d, J=10.1 \mathrm{~Hz})$ & $5.53(1 \mathrm{H}, d, J=10.0 \mathrm{~Hz})$ & $5.52(1 \mathrm{H}, d, J=10.0 \mathrm{~Hz})$ \\
\hline $4^{\prime \prime}$ & $6.56(1 \mathrm{H}, d, J=10.1 \mathrm{~Hz})$ & $6.58(1 \mathrm{H}, d, J=10.1 \mathrm{~Hz})$ & $6.56(1 \mathrm{H}, d, J=10.1 \mathrm{~Hz})$ & $6.62(1 \mathrm{H}, d, J=10.1 \mathrm{~Hz})$ & $6.59(1 \mathrm{H}, d, J=10.1 \mathrm{~Hz})$ \\
\hline $1^{\prime \prime \prime}$ & $3.20(2 \mathrm{H}, \mathrm{m})$ & & & $3.13(2 \mathrm{H}, d, J=7.2 \mathrm{~Hz})$ & \\
\hline $2^{\prime \prime \prime}$ & $5.23(1 \mathrm{H}, \mathrm{m})$ & $4.31(1 \mathrm{H}, t, J=8.0 \mathrm{~Hz})$ & $4.22(1 \mathrm{H}, \mathrm{m})$ & $5.15(1 \mathrm{H}, t, J=7.2 \mathrm{~Hz})$ & \\
\hline \multirow[t]{2}{*}{$3^{\prime \prime \prime}$} & & $\begin{array}{l}2.87(1 \mathrm{H}, d d, J=8.6 \\
14.9 \mathrm{~Hz})\end{array}$ & $\begin{array}{l}2.88(1 \mathrm{H}, d d, J=8.5 \\
14.2 \mathrm{~Hz})\end{array}$ & & $5.48(1 \mathrm{H}, d, J=9.8 \mathrm{~Hz})$ \\
\hline & & $\begin{array}{l}2.66(1 \mathrm{H}, d d, J=2.0 \\
14.6 \mathrm{~Hz})\end{array}$ & $\begin{array}{l}2.68(1 \mathrm{H}, d d, J=2.9 \\
14.0 \mathrm{~Hz})\end{array}$ & & \\
\hline $4^{\prime \prime \prime}$ & & & & & $6.18(1 \mathrm{H}, d, J=9.8 \mathrm{~Hz})$ \\
\hline $5^{\prime \prime \prime}$ & & $\begin{array}{l}4.98(1 \mathrm{H}, \mathrm{m}) ; 4.86 \\
(1 \mathrm{H}, \mathrm{m})\end{array}$ & $\begin{array}{l}4.91(1 \mathrm{H}, \mathrm{m}) ; 4.78 \\
(1 \mathrm{H}, \mathrm{m})\end{array}$ & & \\
\hline \multirow[t]{2}{*}{ 2"-Me } & $1.44(3 \mathrm{H}, \mathrm{s})$ & $1.43(3 \mathrm{H}, \mathrm{s})$ & $1.43(3 \mathrm{H}, \mathrm{s})$ & $1.46(3 \mathrm{H}, \mathrm{s})$ & $1.40(3 \mathrm{H}, \mathrm{s})$ \\
\hline & $1.42(3 \mathrm{H}, \mathrm{s})$ & $1.43(3 \mathrm{H}, \mathrm{s})$ & $1.41(3 \mathrm{H}, \mathrm{s})$ & $1.44(3 \mathrm{H}, \mathrm{s})$ & $1.38(3 \mathrm{H}, \mathrm{s})$ \\
\hline \multirow[t]{2}{*}{$2^{\prime \prime \prime}-\mathrm{Me}$} & & & & & $1.45(3 \mathrm{H}, \mathrm{s})$ \\
\hline & & & & & $1.45(3 \mathrm{H}, \mathrm{s})$ \\
\hline \multirow[t]{2}{*}{ 3"'-Me } & $1.71(3 \mathrm{H}, \mathrm{s})$ & & & $1.67(3 \mathrm{H}, \mathrm{s})$ & \\
\hline & $1.66(3 \mathrm{H}, \mathrm{s})$ & & & $1.60(3 \mathrm{H}, s)$ & \\
\hline 6"'-Me & & $1.79(3 \mathrm{H}, \mathrm{s})$ & $1.78(3 \mathrm{H}, \mathrm{s})$ & & \\
\hline $5-\mathrm{OH}$ & $11.94(1 \mathrm{H}, \mathrm{s})$ & $12.07(1 \mathrm{H}, \mathrm{s})$ & $11.89(1 \mathrm{H}, \mathrm{s})$ & $11.63(1 \mathrm{H}, \mathrm{s})$ & $11.67(1 \mathrm{H}, \mathrm{s})$ \\
\hline 2'-OMe & & & $3.79(3 \mathrm{H}, \mathrm{s})$ & & \\
\hline 4'-OMe & $3.77(3 \mathrm{H}, \mathrm{s})$ & & & & \\
\hline
\end{tabular}


- Table $2{ }^{13} \mathrm{C}$ NMR $\left(150 \mathrm{MHz}, \delta\right.$ in ppm, $\left.\mathrm{CDCl}_{3}\right)$ data for compounds 1-5.

\begin{tabular}{|c|c|c|c|c|c|}
\hline \multirow[t]{2}{*}{ Position } & 1 & 2 & 3 & 4 & 5 \\
\hline & $\delta_{\mathrm{C}}$ (Type) & $\delta_{\mathrm{C}}$ (Type) & $\delta_{\mathrm{C}}$ (Type) & $\delta_{C}$ (Type) & $\delta_{\mathrm{C}}$ (Type) \\
\hline 2 & $69.3\left(\mathrm{CH}_{2}\right)$ & $69.7\left(\mathrm{CH}_{2}\right)$ & $69.2\left(\mathrm{CH}_{2}\right)$ & $74.0(\mathrm{CH})$ & $70.2\left(\mathrm{CH}_{2}\right)$ \\
\hline 3 & $44.9(\mathrm{CH})$ & $45.4(\mathrm{CH})$ & $44.7(\mathrm{CH})$ & $72.9(\mathrm{CH})$ & $105.1(C)$ \\
\hline 4 & 196.8 (C) & 197.1 (C) & $196.6(C)$ & $194.8(C)$ & 185.1 (C) \\
\hline 5 & 159.0 (C) & $159.0(C)$ & $159.0(C)$ & $158.3(C)$ & $161.5(C)$ \\
\hline 6 & $103.1(C)$ & $103.1(\mathrm{C})$ & $103.1(C)$ & $103.2(C)$ & 96.7 (C) \\
\hline 7 & $163.0(C)$ & $162.8(C)$ & $163.2(\mathrm{C})$ & $163.5(C)$ & $159.3(\mathrm{C})$ \\
\hline 8 & $96.0(\mathrm{CH})$ & $96.1(\mathrm{CH})$ & $96.2(\mathrm{CH})$ & $96.6(\mathrm{CH})$ & $103.6(C)$ \\
\hline 9 & $162.3(\mathrm{C})$ & $162.4(C)$ & $162.2(\mathrm{C})$ & $162.4(C)$ & $163.5(C)$ \\
\hline 10 & $101.3(\mathrm{C})$ & $101.8(C)$ & $101.3(\mathrm{C})$ & $101.5(C)$ & $101.3(\mathrm{C})$ \\
\hline $1^{\prime}$ & $113.5(C)$ & 113.9 (C) & $114.0(C)$ & $115.0(C)$ & $140.6(C)$ \\
\hline $2^{\prime}$ & $154.2(C)$ & 154.9 (C) & $158.4(C)$ & 155.9 (C) & $146.6(C)$ \\
\hline $3^{\prime}$ & $100.9(\mathrm{CH})$ & $106.3(\mathrm{CH})$ & $101.1(\mathrm{CH})$ & $105.7(\mathrm{CH})$ & $99.3(\mathrm{CH})$ \\
\hline $4^{\prime}$ & 158.1 (C) & 156.7 (C) & 155.1 (C) & $155.6(C)$ & $148.7(C)$ \\
\hline $5^{\prime}$ & $122.8(C)$ & $118.5(\mathrm{C})$ & $119.5(C)$ & $118.4(C)$ & $114.8(C)$ \\
\hline $6^{\prime}$ & $127.6(\mathrm{CH})$ & $130.4(\mathrm{CH})$ & $129.4(\mathrm{CH})$ & $127.4(\mathrm{CH})$ & $105.9(\mathrm{CH})$ \\
\hline $2^{\prime \prime}$ & 78.5 (C) & 78.5 (C) & $78.6(C)$ & 78.9 (C) & $78.9(C)$ \\
\hline $3^{\prime \prime}$ & $126.2(\mathrm{CH})$ & $126.2(\mathrm{CH})$ & $126.2(\mathrm{CH})$ & $126.5(\mathrm{CH})$ & $126.7(\mathrm{CH})$ \\
\hline $4^{\prime \prime}$ & $115.1(\mathrm{CH})$ & $115.1(\mathrm{CH})$ & $115.0(\mathrm{CH})$ & $114.8(\mathrm{CH})$ & $114.9(\mathrm{CH})$ \\
\hline $1^{\prime \prime \prime}$ & $27.8\left(\mathrm{CH}_{2}\right)$ & & & $28.7\left(\mathrm{CH}_{2}\right)$ & \\
\hline $2^{\prime \prime \prime}$ & $122.6(\mathrm{CH})$ & $78.4(\mathrm{CH})$ & $75.6(\mathrm{CH})$ & $121.3(\mathrm{CH})$ & $76.2(\mathrm{C})$ \\
\hline $3^{\prime \prime \prime}$ & $132.4(\mathrm{CH})$ & $37.4\left(\mathrm{CH}_{2}\right)$ & $36.6\left(\mathrm{CH}_{2}\right)$ & $135.1(C)$ & $128.6(\mathrm{CH})$ \\
\hline $4^{\prime \prime \prime}$ & & $146.5(C)$ & $147.1(\mathrm{C})$ & & $122.0(\mathrm{CH})$ \\
\hline $5^{\prime \prime \prime}$ & & $111.3\left(\mathrm{CH}_{2}\right)$ & $110.6\left(\mathrm{CH}_{2}\right)$ & & \\
\hline \multirow[t]{2}{*}{ 2"-Me } & $28.4\left(\mathrm{CH}_{3}\right)$ & $28.5\left(\mathrm{CH}_{3}\right)$ & $28.5\left(\mathrm{CH}_{3}\right)$ & $28.5\left(\mathrm{CH}_{3}\right)$ & $28.5\left(\mathrm{CH}_{3}\right)$ \\
\hline & $28.4\left(\mathrm{CH}_{3}\right)$ & $28.5\left(\mathrm{CH}_{3}\right)$ & $28.5\left(\mathrm{CH}_{3}\right)$ & $28.4\left(\mathrm{CH}_{3}\right)$ & $28.5\left(\mathrm{CH}_{3}\right)$ \\
\hline \multirow[t]{2}{*}{ 2"'-Me } & & & & & $27.6\left(\mathrm{CH}_{3}\right)$ \\
\hline & & & & & $27.4\left(\mathrm{CH}_{3}\right)$ \\
\hline \multirow[t]{2}{*}{ 3"'-Me } & $25.7\left(\mathrm{CH}_{3}\right)$ & & & $25.5\left(\mathrm{CH}_{3}\right)$ & \\
\hline & $17.7\left(\mathrm{CH}_{3}\right)$ & & & $17.6\left(\mathrm{CH}_{3}\right)$ & \\
\hline 6"'-Me & & $18.1\left(\mathrm{CH}_{3}\right)$ & $18.1\left(\mathrm{CH}_{3}\right)$ & & \\
\hline 2'-OMe & & & $55.5\left(\mathrm{CH}_{3}\right)$ & & \\
\hline 4'-OMe & $55.4\left(\mathrm{CH}_{3}\right)$ & & & & \\
\hline
\end{tabular}

\section{Materials and Methods}

\section{General experiment procedures}

Optical rotations were recorded on an AUTOPOL IV-T automatic polarimeter. The ECD spectra were obtained using a JASCO J-810 Circular Dichroism Spectrometer. All NMR data were obtained using a Bruker Avance III $600 \mathrm{MHz}$ NMR spectrometer, and the MS was obtained using a Thermo Fisher Ultimate 3000 HPLC TOF-MS. Toyopearl HW-40C and Sephadex LH-20 were employed for gel permeation. A macroporous adsorption resin (D101) and silica gel (100-200, 200-300 meshes) were employed for column chromatography. HPLC separations were carried out on a WuFeng LC100 pump that was equipped with an RI2000 refractive index detector using a YMC-Pack ODS-A column $(10 \times 250 \mathrm{~mm}, 5 \mu \mathrm{m})$ and a YMC-Pack SIL column $(10 \times 250 \mathrm{~mm}, 5 \mu \mathrm{m})$. The change of hu- 


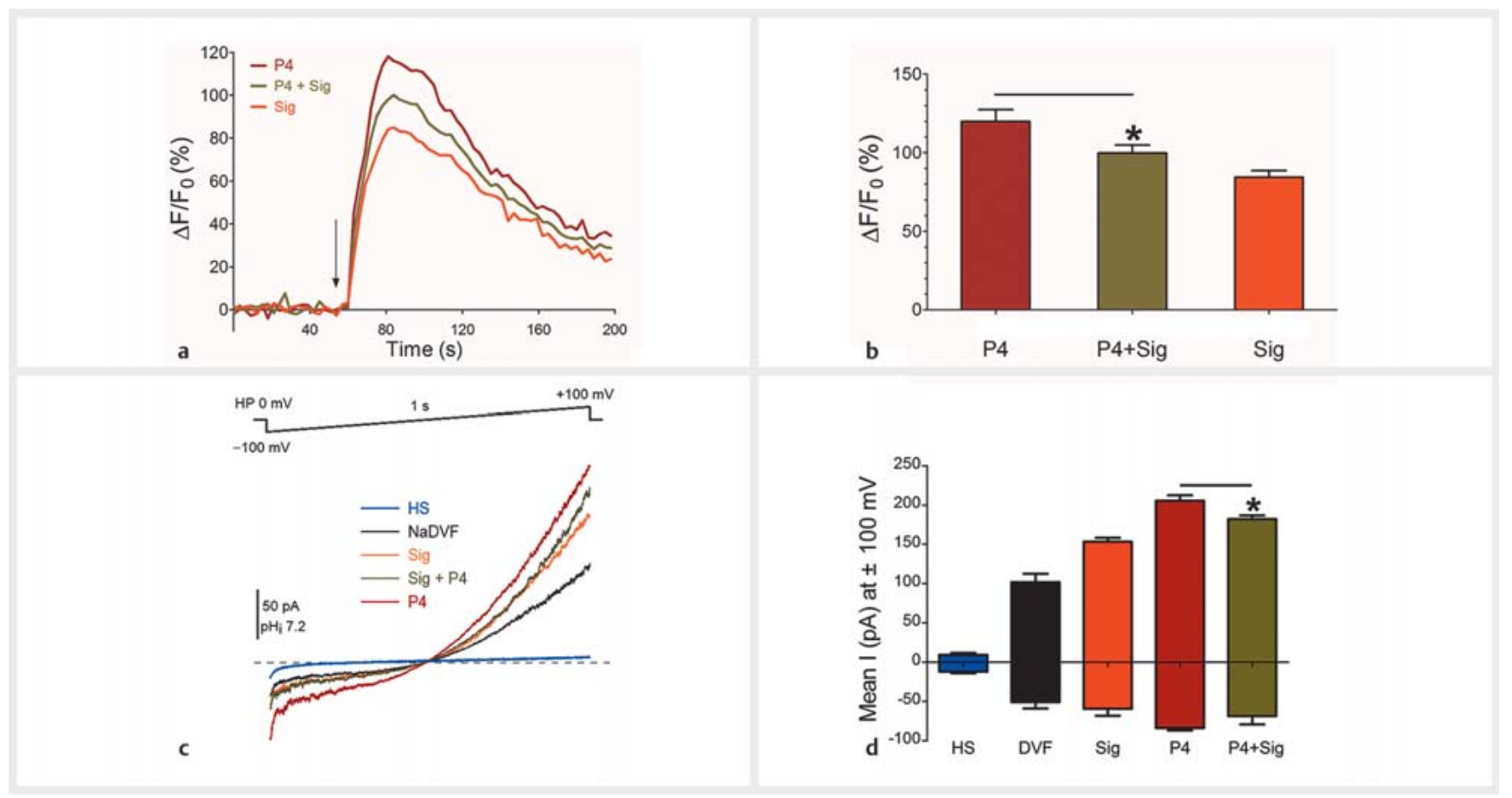

- Fig. 3 Rhynchone A (1) inhibited the activation of human CatSper induced by P4. a The typical fluorescence traces of [Ca $\left.{ }^{2+}\right]_{i}$ signals after exposure to rhynchone A (1), P4, and their mixture. Arrow indicates the time point of additives in human sperm. $b$ Average amplitudes of the $\mathrm{Ca}^{2+}$ response related to a are shown. $\mathrm{c}$ Representative monovalent current of human CatSper after injecting rhynchone A (1), P4, and their mixture. The monovalent CatSper current was recorded in the presence of sodium-based divalent-free solution (NaDVF) by a voltage-clamp ramp protocol (from $-100 \mathrm{mV}$ to $+100 \mathrm{mV}, 1 \mathrm{~s}$ ). Holding potential (HP) was set to $0 \mathrm{mV}$. d Average currents of the CatSper channel at $-100 \mathrm{mV}$ (negative) and $+100 \mathrm{mV}$ (positive) as related to $\mathrm{c}$ are shown. Data are expressed as the mean $\pm \mathrm{SEM} ; \mathrm{n}=4,{ }^{*} \mathrm{p}<0.05$.

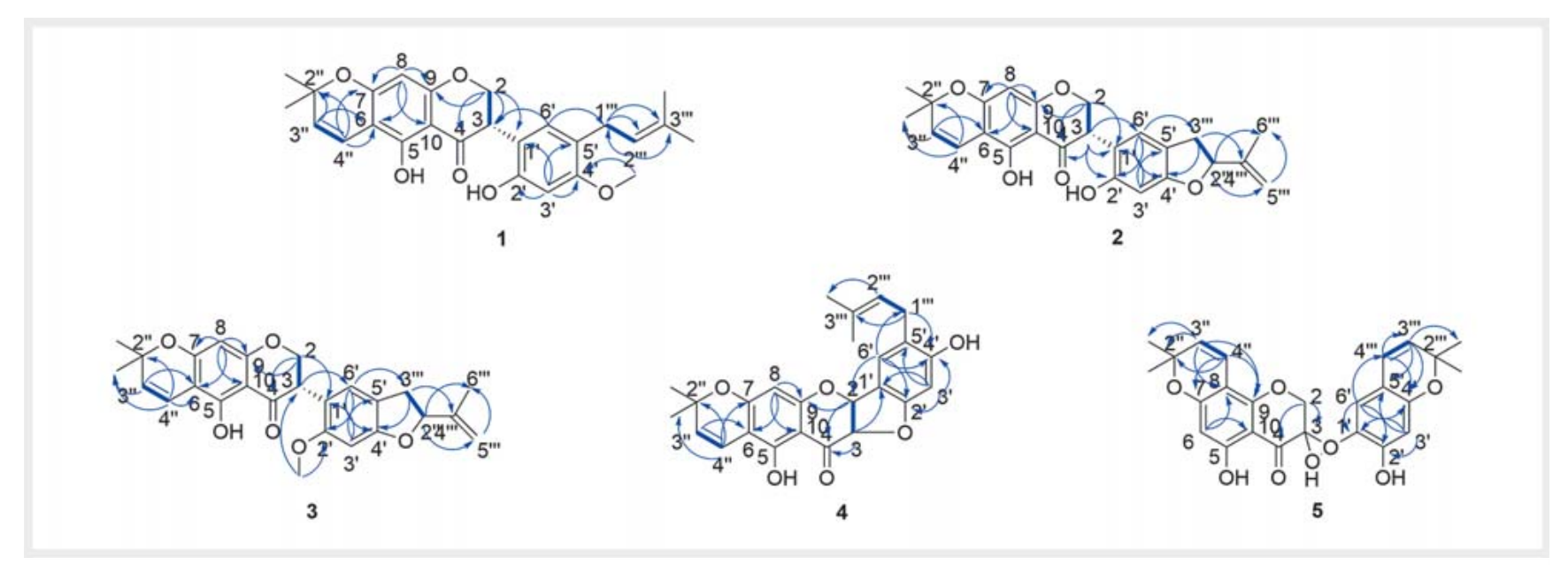

- Fig. 4 Key HMBC and ${ }^{1} \mathrm{H}-{ }^{1} \mathrm{H}$ COSY of rhynchones A-E (1-5).

man sperm $\left[\mathrm{Ca}^{2+}\right]_{i}$ was measured using the fluorescent $\mathrm{Ca}^{2+}$ indicator Fluo-4 AM with the EnSpire Multimode Plate Reader. Pipettes were prepared by a Sutter Micropipette Puller P1000 and Narishige Microforge MF830. The CatSper current was recorded by a patch-clamping system constructed by an Olympus IX71 inverted microscope, a Sutter electric triaxial micromanipulator, Axon Axopatch 200B, and Axon Digidata 1550.

\section{Plant material}

The whole herb of $R$. volubilis was collected in Zaoyang County by Mr. Rui-Zhong Zhou, a pharmacist from Zaoyang Hospital of Traditional Chinese Medicine, Xiangyang City, Hubei Province. The plant was identified by Dr. Jinbo Fang, who is an Associate Professor from the School of Pharmacy, Tongji Medical College of Huazhong University of Science and Technology (China), where the voucher specimens (NO. RVL 20181 101) were deposited. 


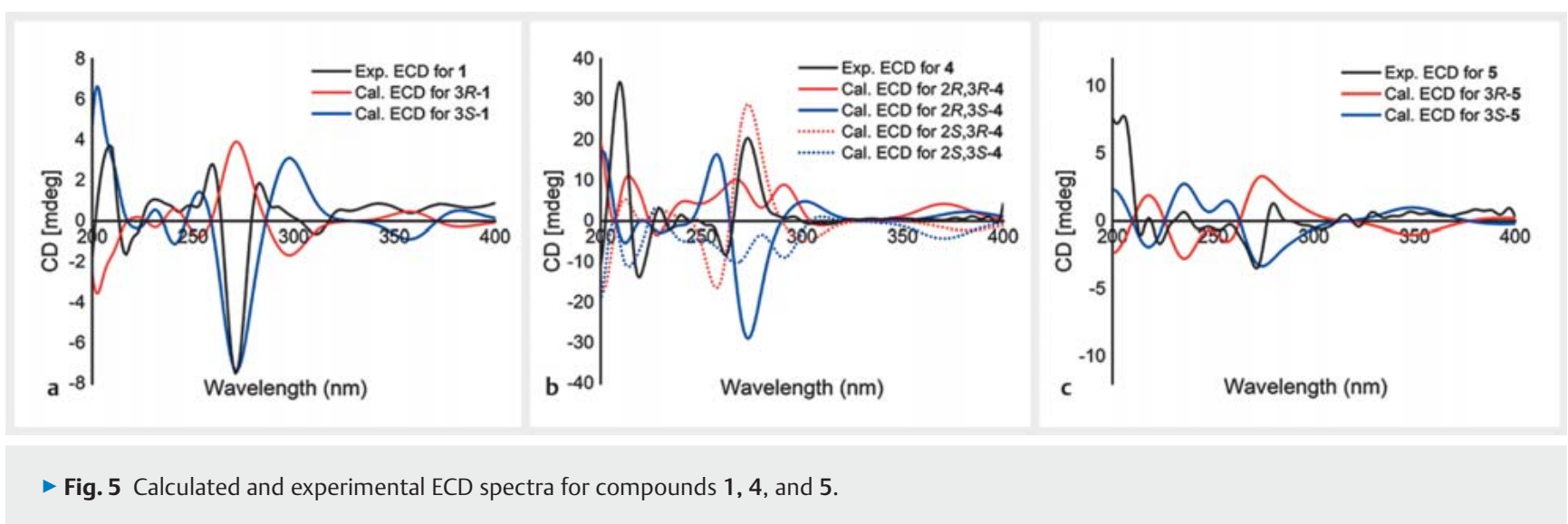

\section{Extraction and isolation}

The air-dried whole plant of $R$. volubilis $(10 \mathrm{~kg})$ was powdered and then extracted three times ( $24 \mathrm{~h}$ each time) with $95 \% \mathrm{EtOH}$ at room temperature to obtain a crude extract after filtration and evaporation of the combined solution. The crude extract was suspended in $\mathrm{H}_{2} \mathrm{O}$ followed by solvent partitions with $\mathrm{PE}$, EtOAc, and $n$ - $\mathrm{BuOH}$, then concentrated in a vacuum to afford extracts weighing $29.1 \mathrm{~g}, 138.8 \mathrm{~g}$, and $179.2 \mathrm{~g}$, respectively.

PE Fr. (28.1 g) was chromatographed on silica gel (100-200 mesh) (PE-EtOAc 100:1, 99:1, 49:1, 19:1, 14:1, 12:1, 9:1, $4: 1,1: 1, v / v)$ to afford nine fractions (Frs. P0101-0109). Fr. P0105 (4.2 g) was subjected to Toyopearl HW-40C $\left(\mathrm{CH}_{2} \mathrm{Cl}_{2}-\right.$ $\mathrm{MeOH}, 2: 1, v / v)$, resulting in six fractions (Frs. P0701-0706). Fr. P0705 (2.2 g) was isolated by RP-C18 $\left(\mathrm{MeOH}-\mathrm{H}_{2} \mathrm{O}, 6: 4,7: 3\right.$, $8: 2,9: 1$ to $1: 0, v / v)$ to get Frs. P0901-0905. Fr. P0904 $(127.4 \mathrm{mg})$ was purified by RP-HPLC $\left(\mathrm{MeOH}-\mathrm{H}_{2} \mathrm{O}, 75: 25,1.5 \mathrm{~mL} /\right.$ $\mathrm{min}$ ) to afford compounds 1 (4.6 mg, $\left.t_{R}=86.2 \mathrm{~min}\right), 2$ (20.7 mg, $\left.t_{R}=91.1 \mathrm{~min}\right)$, and $4\left(4.1 \mathrm{mg}, t_{R}=107.6 \mathrm{~min}\right)$.

Fr. P0106 $(7.9 \mathrm{~g})$ was subjected to RP-C18 $\left(\mathrm{MeOH}-\mathrm{H}_{2} \mathrm{O}, 4: 6\right.$, $5: 5,6: 4,7: 3,8: 2,9: 1$ to $1: 0, v / v)$ to get Frs. P1201-1208. Fr. P1207 (1.8g) was chromatographed on Toyopearl HW-40C $\left(\mathrm{CH}_{2} \mathrm{Cl}_{2}-\mathrm{MeOH}, 2: 1, \mathrm{v} / \mathrm{v}\right)$ and Sephadex $\mathrm{LH}-20(\mathrm{MeOH})$ to obtain Frs. P1801-1805. Fr. P1804 (206.9 mg) was isolated by silica gel (300-400 mesh) eluted with (PE-acetone, 9:1-4:1, v/v), then purified by RP-HPLC and eluted with $\mathrm{MeOH}-\mathrm{H}_{2} \mathrm{O}(86: 14,1.5 \mathrm{~mL} /$ min) followed by PTLC and eluted with PE-acetone $(4: 1)$ to afford 6 (3.6 mg).

EtOAc Fr. (138.8 g) was separated using resin HP-20SS (75$150 \mu \mathrm{m})$ and eluted with $\mathrm{MeOH}-\mathrm{H}_{2} \mathrm{O}(4: 6,6: 4,8: 2,9: 1,0: 10$, $\mathrm{v} / \mathrm{v}$ ) to obtain six fractions (Frs. E0101-0106). Fr. E0103 (54.2 g) was subjected to silica gel [100-200 mesh, $\left(\mathrm{CH}_{2} \mathrm{Cl}_{2}-\mathrm{MeOH}\right.$, 100:0, 99:1, 98:2, 97:3, 96:4, 95:5, 9:1, 8:1, 6:1, 2:1, 1:1, $0: 1, v / v)$ ] to afford 11 fractions (Frs. E0201-0211). Fr. E0206 $(3.8 \mathrm{~g})$ was chromatographed on silica gel $(60 \mu \mathrm{m})$ and eluted with $\mathrm{CH}_{2} \mathrm{Cl}_{2}-\mathrm{MeOH}(200: 1,100: 1,50: 1,1: 1,0: 1, \mathrm{v} / \mathrm{v})$ to get Frs. E0901-0905. Fr. E0902 (829.9 mg) was purified by Sephadex $\mathrm{LH}-20(\mathrm{MeOH})$ followed by RP-HPLC eluted with $\mathrm{MeOH}-\mathrm{H}_{2} \mathrm{O}$ (72:28, $1.5 \mathrm{~mL} / \mathrm{min})$ and PTLC eluted with $\mathrm{CH}_{2} \mathrm{Cl}_{2}-\mathrm{MeOH}$ (49:1, $\mathrm{v} / \mathrm{v})$, to afford compounds $3(4.3 \mathrm{mg}), 5(22.7 \mathrm{mg}), 7$ (4.9 mg), and $8(5.8 \mathrm{mg})$.
Fr. E0104 $(64.7 \mathrm{~g})$ was isolated with silica gel $(60 \mu \mathrm{m})$ and eluted with $n$-hexane-EtOAc, 6:1, 5:1, 4:1, 3:1, 2:1, 0:1, v/v) to afford nine fractions (Frs. E1301-1309). Frs. E1301-1303 $(6.4 \mathrm{~g})$ were chromatographed on Toyopearl HW-40C $\left(\mathrm{CH}_{2} \mathrm{Cl}_{2}\right.$ $\mathrm{MeOH}, 2: 1, \mathrm{v} / \mathrm{v})$ and Sephadex $\mathrm{LH}-20(\mathrm{MeOH})$ and purified by RP-HPLC and eluted with $\mathrm{MeOH}-\mathrm{H}_{2} \mathrm{O}(69: 31,1.5 \mathrm{~mL} / \mathrm{min})$ followed by PTLC and eluted with $\left(\mathrm{CH}_{2} \mathrm{Cl}_{2}-\mathrm{MeOH}, 49: 1, \mathrm{v} / \mathrm{v}\right)$ to afford $14(6.3 \mathrm{mg}), 15(10.7 \mathrm{mg}), 16(6.1 \mathrm{mg})$, and $17(7.7 \mathrm{mg})$. Frs. E1304-1307 (15.2g) were separated using RP-C18 and eluted with $\mathrm{MeOH}-\mathrm{H}_{2} \mathrm{O}(70: 30,75: 25,80: 20,85: 15,90: 10$, $100: 0, v / v)$ to obtain seven fractions (Frs. E1801-1807). Fr. E1804 (6.1 g) was chromatographed on silica gel (300-400 mesh) and eluted with PE-EtOAc $(10: 1,9: 1,8: 1,7: 1,6: 1,5: 1,4: 1$, $3: 1,2: 1,1: 1,0: 1, v / v)$ to afford 11 fractions (Frs. E1901-1911). Fr. E1906 (3.1 g) was successively isolated with Sephadex LH-20 $(\mathrm{MeOH}), \mathrm{RP}-\mathrm{HPLC}$, and eluted with $\mathrm{MeOH}-\mathrm{H}_{2} \mathrm{O}(74: 26,1.5 \mathrm{~mL} /$ min) followed by PTLC and eluted with $\left(\mathrm{CH}_{2} \mathrm{Cl}_{2}-\mathrm{MeOH}, 50: 1\right.$, v/v) to afford $10(15.1 \mathrm{mg}), 11(12.8 \mathrm{mg}), 12(5.2 \mathrm{mg})$, and 13 $(13.6 \mathrm{mg})$. Fr. E1807 (4.7 g) was chromatographed on silica gel $(60 \mu \mathrm{m})$ and eluted with PE-EtOAc $(10: 1,9: 1,8: 1,7: 1,6: 1$, $5: 1,4: 1,3: 1,2: 1,1: 1,0: 1, v / v$ ) to afford 11 fractions (Frs. E2201-2211). Frs. E2204-2205 (958.2 mg) were successively isolated with Sephadex LH-20 (MeOH), RP-HPLC, and eluted with $\mathrm{MeOH}-\mathrm{H}_{2} \mathrm{O}(76: 24,1.5 \mathrm{~mL} / \mathrm{min})$ followed by PTLC and eluted with PE-acetone $(4: 1)$ to afford $9(7.0 \mathrm{mg})$ and $18(6.6 \mathrm{mg})$.

\section{Quantum chemistry calculations}

A conformational search of the compounds was implemented in Maestro 10.2 software (Schrodinger, LLC) where conformers with Boltzmann populations $>5 \%$ were taken into further quantum chemistry calculations. The geometry optimizations, frequency analysis, and TD-DFT calculations of each conformer were subsequently carried out using the B3LYP/6-31 g (d, p) level with Gaussian 16 B.01 [34]. The solvent effects of methanol were taken into consideration by using a solvation model of IEFPCM during the calculations [35]. The calculated ECD data were Boltzmann averaged according to Gibbs free energy and their ECD spectra were generated by the SpecDis v1.71 program [38] with a bandwidth $(\sigma)$ of $0.16 \mathrm{eV}$. For all calculated spectra, the vertical axes were scaled to fit the experimental spectra. The wavelength shift 
of 2, 0 , and $-35 \mathrm{~nm}$ was employed for 1,4 , and 5 , respectively (Fig. 41-43S, Supporting Information).

\section{Measurement of sperm $\left[\mathrm{Ca}^{2+}\right]_{\mathrm{i}}$}

The change of human sperm $\left[\mathrm{Ca}^{2+}\right]_{i}$ was measured using the fluorescent $\mathrm{Ca}^{2+}$ indicator Fluo-4 AM with the EnSpire Multimode Plate Reader as previously described [39]. The action of compounds 1-18 (100 mM stock in DMSO) on $\left[\mathrm{Ca}^{2+}\right]_{i}$ of human sperm was detected. The final concentration of DMSO was $0.1 \%$. The change of sperm $\left[\mathrm{Ca}^{2+}\right]_{i}$ was calculated by $\Delta \mathrm{F} / \mathrm{F}_{0}(\%)$, indicating the percent $(\%)$ of fluorescent changes $(\Delta \mathrm{F})$ normalized to the mean basal fluorescence before the application of any chemicals $\left(F_{0}\right) . \Delta F / F_{0}(\%)=\left(F-F_{0}\right) / F_{0} \times 100 \%$, where $F$ indicates the fluorescent intensity at each recorded time point.

\section{Compounds assay - sperm patch-clamp recordings}

The whole-cell patch-clamp technique was applied to record human sperm CatSper as previously described [40]. Seals were formed at the sperm cytoplasmic droplet or the neck region by a 15-30 $\mathrm{M} \Omega$ pipette. The transition into whole-cell mode was then made by applying short (1 ms) voltage pulses (400-650 mV) combined with light suction. The currents were stimulated by $1 \mathrm{~s}$ voltage ramps from -100 to $+100 \mathrm{mV}$ from a holding potential of $0 \mathrm{mV}$. The monovalent current of CatSper and divalent-free (DVF) solution (150 mM NaCl, $20 \mathrm{mM}$ HEPES, and $5 \mathrm{mM}$ EDTA, $\mathrm{pH}$ 7.4) was used to record basal CatSper monovalent currents. Then, 1,10 , and $100 \mu \mathrm{M}$ compounds (1-18), $1 \mu \mathrm{M}$ progesterone, and $100 \mu \mathrm{M}$ compounds (1-18) together with $1 \mu \mathrm{M}$ progesterone in DVF were perfused to record CatSper currents. Data were analyzed with Clampfit version 10.4 software.

\section{Rhynchone A (1)}

Pale yellow solid; $[\alpha]_{\mathrm{D}}^{20}-5.33^{\circ}\left(\mathrm{c} 0.1, \mathrm{CH}_{3} \mathrm{OH}\right)$; UV $(\mathrm{MeOH}) \lambda_{\max } \mathrm{nm}$ ( $\log \varepsilon): 204$ (4.02), 272 (3.97). IR (KBr) $v_{\max }$ 3423, 2970, 2881, 1639, 1560, 1494, 1392, $1187 \mathrm{~cm}^{-1}$; ${ }^{1} \mathrm{H}$ NMR $\left(600 \mathrm{MHz}\right.$ in $\left.\mathrm{CDCl}_{3}\right)$ and ${ }^{13} \mathrm{C}$ NMR $\left(150 \mathrm{MHz}\right.$ in $\left.\mathrm{CDCl}_{3}\right)$, for data, see $>$ Tables 1 and 2; HR-ESI-TOF-MS [M- H] $]^{-}$m/z 435.1794 ([M - H] $]^{-}$calcd. 435.1807).

\section{Rhynchone B (2)}

Yellow oil; $[\alpha]_{\mathrm{D}}^{20}-16.7^{\circ}$ (c $\left.0.1, \mathrm{CH}_{3} \mathrm{OH}\right)$; UV $(\mathrm{MeOH}) \lambda_{\max } \mathrm{nm}(\log$ ع): 203 (3.68), 272 (3.59). IR ( $\mathrm{KBr}) v_{\max } 3436,2982,2881,2382$, 1624, 1555, 1397, $1165 \mathrm{~cm}^{-1}$; ${ }^{1} \mathrm{H}$ NMR $\left(600 \mathrm{MHz}\right.$ in $\left.\mathrm{CDCl}_{3}\right)$ and ${ }^{13} \mathrm{C} \mathrm{NMR}\left(150 \mathrm{MHz}\right.$ in $\left.\mathrm{CDCl}_{3}\right)$, for data, see $>$ Tables 1 and 2; HRESI-TOF-MS [M + $\left.\mathrm{H}_{2} \mathrm{O}-\mathrm{H}\right]^{-} \mathrm{m} / \mathrm{z} 437.1606\left(\left[\mathrm{M}+\mathrm{H}_{2} \mathrm{O}-\mathrm{H}\right]^{-}\right.$calcd. 437.1600).

\section{Rhynchone C (3)}

Yellow powder; $[\alpha]_{\mathrm{D}}^{20}-23.1^{\circ}$ (c $\left.0.1, \mathrm{CH}_{3} \mathrm{OH}\right)$; UV $(\mathrm{MeOH}) \lambda_{\max } \mathrm{nm}$ ( $\log \varepsilon): 202$ (3.66), 272 (3.54). IR (KBr) $v_{\max } 3441,2980,2882$, 1644, 1627, 1392, $1315 \mathrm{~cm}^{-1} ;{ }^{1} \mathrm{H}$ NMR $\left(600 \mathrm{MHz}\right.$ in $\left.\mathrm{CDCl}_{3}\right)$ and ${ }^{13} \mathrm{C}$ NMR $\left(150 \mathrm{MHz}\right.$ in $\left.\mathrm{CDCl}_{3}\right)$, for data, see - Tables 1 and 2; HRESI-TOF-MS [M- H] $]^{-}$m/z 435.1794 ([M - H] $]^{-}$calcd. 435.1807).

\section{Rhynchone D (4)}

Yellow oily solid; $[\alpha]_{\mathrm{D}}^{20}-15.3^{\circ}$ (c 0.1, $\left.\mathrm{CH}_{3} \mathrm{OH}\right)$; UV $(\mathrm{MeOH}) \lambda_{\max } \mathrm{nm}$ ( $\log \varepsilon$ ): 203 (3.98), 226 (3.56), 273 (3.85). IR (KBr) $v_{\max } 3342$, 2980, 1630, 1627, 1491, 1376, 1363, 1169, 1130, $1097 \mathrm{~cm}^{-1} ;{ }^{1} \mathrm{H}$
NMR $\left(600 \mathrm{MHz}\right.$ in $\mathrm{CDCl}_{3}$ ) and ${ }^{13} \mathrm{C} \mathrm{NMR}\left(150 \mathrm{MHz}\right.$ in $\left.\mathrm{CDCl}_{3}\right)$, for data, see - Tables 1 and 2; HR-ESI-TOF-MS [M + $\left.\mathrm{H}_{2} \mathrm{O}-\mathrm{H}\right]^{-} \mathrm{m} / \mathrm{z}$ $437.1639\left(\left[\mathrm{M}+\mathrm{H}_{2} \mathrm{O}-\mathrm{H}\right]^{-}\right.$calcd. 437.1600).

\section{Rhynchone E (5)}

Yellow oily solid; $[\alpha]_{\mathrm{D}}^{20}-67.1^{\circ}\left(\mathrm{c} 0.1, \mathrm{CH}_{3} \mathrm{OH}\right)$; UV $(\mathrm{MeOH}) \lambda_{\max } \mathrm{nm}$ ( $\log \varepsilon): 212$ (3.90), 276 (3.92), 322 (3.76). IR (KBr) $v_{\max } 3440$, 2980, 2881, 1647, 1627, 1484, 1381, $1145 \mathrm{~cm}^{-1} ;{ }^{1} \mathrm{H}$ NMR $\left(600 \mathrm{MHz}\right.$ in $\left.\mathrm{CDCl}_{3}\right)$ and ${ }^{13} \mathrm{C} \mathrm{NMR}\left(150 \mathrm{MHz}\right.$ in $\left.\mathrm{CDCl}_{3}\right)$, for data, see $>$ Tables 1 and 2; HR-ESI-TOF-MS [M- H] $]^{-} \mathrm{m} / \mathrm{z} 451.1366$ $\left([\mathrm{M}-\mathrm{H}]^{-}\right.$calcd. 451.1392).

\section{Supporting information}

HR-ESI-MS, NMR spectra, and ECD of compounds 1-5, and effect of extracts and compounds 1-18 on human sperm $\left[\mathrm{Ca}^{2+}\right]_{i}$ are available as Supporting Information.

\section{Contributors' Statement}

J. Xiang: investigation, visualization, and writing - original draft. $H$. Kang: investigation, visualization, and writing - original draft. H. G. Li: resources and funding acquisition. Y.L. Shi: investigation, visualization, and revision. Y.L. Zhang: investigation. C.L. Ruan: investigation. L.H. Liu: investigation. H.Q. Gao: investigation. T. Luo: resources and funding acquisition. G.S. Hu: investigation. W. L. Zhu: supervision. J. M. Jia: supervision. J. C. Chen: resources. J. B. Fang: writing - review and editing, and funding acquisition. All authors approved the final version of the manuscript.

\section{Acknowledgements}

This research was financially supported by the National Key Research and Development Plan (No. 2016YFC1 000 905), the National Natural Science Foundation (No. 31000150 ), the Fundamental Research Funds for the Central Universities (No. HUST2016YXMS145), the Special Funds for Central Government to Guide Local Scientific and Technological Development (No. 20202ZDB01 013), and the Open Funds of State Key Laboratory of Magnetic Resonance and Atomic and Molecular Physics (No. T152602). We appreciate Dr. Zhijian Xu and Bo Li, CAS Key Laboratory of Receptor Research \& Drug Discovery and Design Center, Shanghai Institute of Materia Medica, Chinese Academy of Sciences, for their help with the computational calculations.

\section{Conflict of Interest}

The authors declare that they have no conflict of interest.

\section{References}

[1] Editorial Committee of Flora of China, Chinese Academy of Sciences. Rhynchosia Loureiro, Fl. Cochinch. 2: 425, 460. 1790, nom. cons. Beijing: Beijing Science Press; 2010: 5

[2] Guan HT, Fang F, Xiong Z, Meng TQ, Huang SX. n-Butanol extract of Rhynchosia volubilis Lour: a potent spermicidal agent In Vitro. J Huazhong Univ Sci Technolog Med Sci 2014; 34: 398-402

[3] Xiang J, Ruan CL, Liu D, Liu D, Dai YW, Li HG, Fang JB. Chemical constituents from Rhynchosia volubilis. Chin Tradit Herbal Drugs 2020; 51: 100 106

[4] Coronado-Aceves EW, Gigliarelli G, Garibay-Escobar A, Zepeda RER, Curini M, Cervantes JL, Espitia-Pinzon Cll, Superchi S, Vergura S, Marcotullio MC. New Isoflavonoids from the extract of Rhynchosia precatoria 
(Humb. \& Bonpl. ex Willd.) DC. and their antimycobacterial activity. J Ethnopharmacol 2017; 206: 92-100

[5] Ogungbe IV, Hill GM, Crouch RA, Vogler B, Nagarkoti M, Haber WA, Setzer WN. Prenylated isoflavonoids from Rhynchosia edulis. Nat Prod Commun 2011; 6: 1637-1644

[6] Rammohan A, Gunasekar D, Reddy NV, Vijaya T, Deville A, Bodo B. Structure elucidation and antioxidant activity of the phenolic compounds from Rhynchosia suaveolens. Nat Prod Commun 2015; 10: 609-611

[7] Ahmed W, Ahmad Z, Malik A. Stigmasteryl galactoside from Rhynchosia minima. Phytochemistry 1992; 31: 4038-4039

[8] Wang JG, Xiong CL, Wang SY, Wu YP, Zhang ZH. Comparison of the antifertility effects of four extracts from the roots of Rhynchosia volubilis Lour. Zhonghua Nan Ke Xue 2007; 13: 871-875

[9] Bakshu LM, Venkata Raju RR. Antimicrobial activity of Rhynchosia beddomei. Fitoterapia 2001; 72: 579-582

[10] Bethu MS, Netala VR, Domdi L, Tartte V, Janapala VR. Potential anticancer activity of biogenic silver nanoparticles using leaf extract of Rhynchosia suaveolens: an insight into the mechanism. Artif Cell Nanomed B 2018; 46: 104-114

[11] Jia XJ, Zhang C, Bao JL, Wang K, Tu YB, Wan JB, He CW. Flavonoids from Rhynchosia minima root exerts anti-inflammatory activity in lipopolysaccharide-stimulated RAW 264.7 cells via MAPK/NF-kappa B signaling pathway. Inflammopharmacology 2020; 28: 289-297

[12] Kinjo J, Nagao T, Tanaka T, Nonaka G, Okabe H. Antiproliferative constituents in the plant 8. Seeds of Rhynchosia volubilis. Biol Pharm Bull 2001; 24: $1443-1445$

[13] Kang SA, Jang KH, Cho Y, Jang EK, Ahn DK, Park SK. Antihyperlipidemic effects of black bean (Yak-Kong, Rhynchosia molubilis) and soybean in an ovariectomized rat model. J Nutr 2004; 134: 1275s

[14] Rammohan A, Reddy GM, Bhaskar BV, Gunasekar D, Zyryanov GV. Phytochemistry and pharmacological activities of the genus Rhynchosia: a comprehensive review. Planta 2019; 251: 9

[15] Alasmari W, Costello S, Correia J, Oxenham SK, Morris J, Fernandes L, Ramalho-Santos J, Kirkman-Brown J, Michelangeli F, Publicover S, Barratt CLR. $\mathrm{Ca}^{2+}$ signals generated by CatSper and $\mathrm{Ca}^{2+}$ stores regulate different behaviors in human sperm. J Biol Chem 2013; 288: 6248-6258

[16] Publicover S, Harper CV, Barratt C. [ $\left.\mathrm{Ca}^{2+}\right](\mathrm{i})$ signalling in sperm - making the most of what you've got. Nat Cell Biol 2007; 9: 235-242

[17] Lishko PV, Botchkina IL, Kirichok Y. Progesterone activates the principal $\mathrm{Ca}^{2+}$ channel of human sperm. Nature 2011; 471: 387-391

[18] Kirichok Y, Navarro B, Clapham DE. Whole-cell patch-clamp measurements of spermatozoa reveal an alkaline-activated $\mathrm{Ca}^{2+}$ channel. Nature 2006; 439: 737-740

[19] Brown SG, Publicover S], Barratt CLR, Ramalingam M, Drew E, Publicover SJ, Barratt CLR, Da Silva SM. Human sperm ion channel (dys)function: implications for fertilization. Hum Reprod Update 2019; 25: 758-776

[20] Lishko PV, Botchkina IL, Kirichok Y. Progesterone activates the principal $\mathrm{Ca}^{2+}$ channel of human sperm. Nature 2011; 471: 387-391

[21] Zhang XN, Kang H, Peng LZ, Song DD, Jiang X, Li YT, Chen HY, Zeng XH. Pentachlorophenol inhibits CatSper function to compromise progesterone's action on human sperm. Chemosphere 2020; 259: 127493

[22] Antus S, Gottsegen A, Kolonits P, Nogradi M. Synthesis of rac-5'-O-methylphaseollinisoflavan. Liebigs Ann Chem 1986; 1986: 2179-2181

[23] Deng YH, Xu KP, Zhou Y], Li FS, Zeng GY, Tan GS. A new flavonol from Sophora tonkinensis. J Asian Nat Prod Res 2007; 9: 45-48

[24] Thongnest S, Lhinhatrakool T, Wetprasit N, Sutthivaiyakit P, Sutthivaiyakit S. Eriosema chinense: a rich source of antimicrobial and antioxidant flavonoids. Phytochemistry 2013; 96: 353-359
[25] Ni G, Zhang Q], Wang YH, Chen RY, Zheng ZF, Yu DQ. Chemical constituents of the stem bark of Morus cathayana. J Asian Nat Prod Res 2010; 12: 505-515

[26] Awouafack MD, Spiteller P, Lamshoft M, Kusari S, Ivanova B, Tane P, Spiteller M. Antimicrobial isopropenyl-dihydrofuranoisoflavones from Crotalaria lachnophora. J Nat Prod 2011; 74: 272-278

[27] Bankeu JJK, Khayala R, Lenta BN, Noungoue DT, Ngouela SA, Mustafa SA, Asaad K, Choudhary MI, Prigge ST, Hasanov R, Nkengfack AE, Tsamo E, Ali MS. Isoflavone dimers and other bioactive constituents from the figs of Ficus mucuso. J Nat Prod 2011; 74: 1370-1378

[28] Shao TM, Li XB, Qi CC, Chen GY, Song XP, Han CR, Zheng C]. Chemical constituents of isoflavonoids from roots of Ficus auriculata. Chinese J Org Chem 2018; 38: 710-714

[29] Lin Y, Kuang Y, Li K, Wang S, Song W, Qiao X, Sabir G, Ye M. Screening for bioactive natural products from a 67-compound library of Glycyrrhiza inflata. Bioorgan Med Chem 2017; 25: 3706-3713

[30] Ateba SB, Njamen D, Ukowitz K, Zehl M, Kahlig H, Hobiger S, Jungbauer A, Krenn L. New flavonoids from the underground parts of Eriosema laurentii. Phytochem Lett 2016; 18: 144-149

[31] Zhao SH, Zhang LP, Gao P, Shao ZY. Isolation and characterisation of the isoflavones from sprouted chickpea seeds. Food Chem 2009; 114: 869873

[32] Khan RA, Kapil RS. A facile synthesis of biogenetic precursor, puerarone, isolated from Pueraria sp. J Heterocycl Chem 2001; 38: 1007-1009

[33] Strong AL, Jiang Q, Zhang Q, Zheng SL, Boue SM, Elliott S, Burow ME, Bunnell BA, Wang GD. Design, synthesis, and osteogenic activity of daidzein analogs on human mesenchymal stem cells. ACS Med Chem Lett 2014; 5: 143-148

[34] Frisch M], Trucks GW, Schlegel HB, Scuseria GE, Robb MA, Cheeseman JR, Scalmani G, Barone V, Petersson GA, Nakatsuji H, Li X, Caricato M, Marenich AV, Bloino J, Janesko BG, Gomperts R, Mennucci B, Hratchian HP, Ortiz JV, Izmaylov AF, Sonnenberg JL, Williams-Young D, Ding F, Lipparini F, Egidi F, Goings J, Peng B, Petrone A, Henderson T, Ranasinghe D, Zakrzewski VG, Gao J, Rega N, Zheng G, Liang W, Hada M, Ehara M, Toyota K, Fukuda R, Hasegawa J, Ishida M, Nakajima T, Honda Y, Kitao O, Nakai H, Vreven T, Throssell K, Montgomery JAJ, Peralta JE, Ogliaro F, Bearpark MJ, Heyd J], Brothers EN, Kudin KN, Staroverov VN, Keith TA, Kobayashi R, Normand J, Raghavachari K, Rendell AP, Burant JC, lyengar SS, Tomasi J, Cossi M, Millam JM, Klene M, Adamo C, Cammi R, Ochterski JW, Martin RL, Morokuma K, Farkas O, Foresman JB, Fox DJ. Gaussian 16, Revision B.01. GaussView 5.0. E. U. A. Wallingford, CT: Gaussian Inc.; 2016

[35] Cances E, Mennucci B, Tomasi ]. A new integral equation formalism for the polarizable continuum model: theoretical background and applications to isotropic and anisotropic dielectrics. J Chem Phys 1997; 107: 3032-3041

[36] Slade D, Ferreira D, Marais JPJ. Circular dichroism, a powerful tool for the assessment of absolute configuration of flavonoids. Phytochemistry 2005; 66: 2177-2215

[37] Sun QH, Chou GX. Isoflavonoids from Crotalaria albida inhibit adipocyte differentiation and lipid accumulation in 3T3-L1 cells via suppression of PPAR-gamma pathway. PLoS One 2015; 10: e0135893

[38] Bruhn T, Schaumloffel A, Hemberger Y, Bringmann G. SpecDis: quantifying the comparison of calculated and experimental electronic circular dichroism spectra. Chirality 2013; 25: 243-249

[39] Zou QX, Peng Z, Zhao Q, Chen HY, Cheng YM, Liu Q, He YQ, Weng SQ, Wang HF, Wang T, Zheng LP, Luo T. Diethylstilbestrol activates CatSper and disturbs progesterone actions in human spermatozoa. Hum Reprod 2017; 32: 290-298

[40] Lishko PV, Botchkina IL, Kirichok Y. Progesterone activates the principal $\mathrm{Ca}^{2+}$ channel of human sperm. Nature 2011; 471: 387-391 